\title{
Distributed multipoles from a robust basis-space implementation of the iterated stockholder atoms procedure
}

\author{
Alston J. Misquitta, ${ }^{1}$ Anthony J. Stone, ${ }^{2}$ and Farhang Fazeli ${ }^{1}$ \\ ${ }^{l}$ School of Physics and Astronomy, Queen Mary, University of London, London E1 4NS, U.K. \\ ${ }^{2}$ University Chemical Laboratory, Lensfield Road, Cambridge, CB2 1EW, U.K.
}

(Dated: September 10, 2018)

\begin{abstract}
The recently developed iterated stockholder atoms (ISA) approach of Lillestolen and Wheatley (Chem. Commun. 2008, 5909 (2008)) offers a powerful method for defining atoms in a molecule. However, the real-space algorithm is known to converge very slowly, if at all. Here we present a robust, basis-space algorithm of the ISA method and demonstrate its applicability on a variety of systems. We show that this algorithm exhibits rapid convergence (taking around 10-80 iterations) with the number of iterations needed being unrelated to the system size or basis set used. Further, we show that the multipole moments calculated using this basis-space ISA method are as good as, or better than those obtained from Stone's distributed multipole analysis (J. Chem. Theory Comput. 1, 1128 (2005)), exhibiting better convergence properties and resulting in better behaved penetration energies. This can have significant consequences in the development of intermolecular interaction models.

PACS numbers: 34.20.Gj 31.15.ap 31.15.-p
\end{abstract}

\section{INTRODUCTION}

The concept of atoms in a molecule (AIM) underlies much of our scientific understanding and almost all classical models of atomic and molecular interactions. Almost all force fields are built up from pair-wise atomatom interactions. Many-body non-additive effects, when they are included, are generally added as a correction to this atom-atom picture. This viewpoint has its limitations: it is inappropriate for metals, and gets progressively more inadequate as the electron delocalization length increases [1]. Nevertheless, it is likely that even for these systems, atom-atom models may be useful when augmented with continuum models that account for the metallic component.

The problem with the AIM model is that there is no unique way to define an atom in a molecule. While some atomic properties (e.g., spectral transitions) may be preserved upon chemical bonding, others (e.g., atomic size and charge) are lost or alter dramatically. Some of these are not physical observables, and methods of defining and calculating them remain controversial.

Many techniques for identifying atoms within molecules are concerned only with determining point charges that reproduce the electrostatic potential of the molecule reasonably accurately [e.g., 2, 3]. We are concerned here with obtaining a well-defined specification of an atom within a molecule that provides an accurate and rapidly-converging multipole expansion of the electrostatic field around the atom. For this, we need a description of each atom that is as nearly as possible spherical. This condition rules out AIM approaches such as that of Bader [4], which is very well defined and has some useful properties, but which leads to highly non-spherical atomic shapes that result in multipole expansions with poor convergence properties [5-7]. Of the AIM methods that result in nearly-spherical atoms, the Hirshfeld stockholder method is one of the most popular. In this method, given functions $w^{a}(\mathbf{r})$ describing the spherically-averaged electron density of the free atoms, we define the atom in the molecule $\rho^{a}(\mathbf{r})$ using:

$$
\rho^{a}(\mathbf{r})=\rho(\mathbf{r}) \times \frac{w^{a}(\mathbf{r})}{\sum_{b} w^{b}(\mathbf{r})},
$$

where $\rho$ is the total molecular density and the indices $a$ and $b$ label the atoms. However this method has two disadvantages: (1) it requires pre-computed shape functions obtained from free atom calculations, and (2) as a result, it is unable to respond to changes in the atomic densities due to chemical bonding. The last point is subtle and requires an explanation. A free carbon atom is more diffuse than a free oxygen atom and this difference is reflected in the Hirshfeld shape functions of these atoms. However, on formation of the $\mathrm{C}-\mathrm{O}$ bond, say in carbon dioxide, the carbon atom must be more compact and the oxygens more diffuse, as the more electronegative oxygen atoms draw some of the electronic density away from the carbon atom. The Hirshfeld procedure does not take account of this phenomenon.

Recently, Lillestolen and Wheatley [8] proposed a novel and rather appealing alternative to the conventional Hirshfeld procedure. In their iterated stockholder atoms (ISA) method no free-atom shape functions $w^{a}(\mathbf{r})$ are needed. Instead, all we do is assume the existence of these spherically symmetric shapes that are, by definition, required to be spherical averages of the atomic densities defined in eqn. (1), that is,

$$
w^{a}(\mathbf{r})=\left\langle\rho^{a}(\mathbf{r})\right\rangle_{\mathrm{sph}},
$$

where the angle bracket indicates the spherical average. The idea here is to make an initial guess for the shape 
functions $w^{a}$, with the only restrictions on them being that they are integrable and positive over all space, and then to iterate eqns. (1) and (2) until the shape functions attain a desired convergence.

Using a real-space implementation of the ISA algorithm, Lillestolen and Wheatley showed that this scheme always converges to a unique solution, and that the atomic charges obtained from the ISA method appear to reproduce the molecular dipole moments better than other distribution schemes. This is promising; if it is generally true, the nearly-spherical ISA atoms could be useful for the construction of compact distributed multipole schemes for molecular systems. If this were the only feature of the ISA atoms, this method would be no different from the standard Hirshfeld technique. However, as we shall see, the ISA atoms additionally capture changes that we commonly associate with chemical bonding. This effect is totally absent in the usual Hirshfeld approach.

The ISA partitioning method is not limited to the density only, but, in a straightforward generalization, can be used to obtain distributed second-order quantities such as the frequency-dependent polarizabilities. However, to achieve this, we cannot use a real-space, gridbased ISA algorithm due to the relatively large computational cost of grid-based methods, and the prohibitively large number of transition densities we would have to partition. Consequently we need a basis-space implementation. A further motivation for this is that, although the Lillestolen-Wheatley method is guaranteed to converge, in practice it is found to converge very slowly[9].

\section{THEORY AND NUMERICAL DETAILS}

The basic idea in the basis-space approach is to use expansions for all the quantities that appear in eqns. (1) and (2). Our goal is to construct an appropriate functional that allows us to obtain the expansion coefficients. The density will normally be expanded in an auxiliary basis set using standard density-fitting techniques:

$$
\tilde{\rho}(\mathbf{r})=\sum_{k} d_{k} \chi_{k}(\mathbf{r})
$$

Here, $d_{k}$ are the expansion coefficients and $\chi_{k}$ the auxiliary basis set. The density fitting is performed by minimizing the functional

$$
\Delta^{\mathrm{DF}}=\iint(\rho(\mathbf{r})-\tilde{\rho}(\mathbf{r})) \frac{1}{\left|\mathbf{r}-\mathbf{r}^{\prime}\right|}\left(\rho\left(\mathbf{r}^{\prime}\right)-\tilde{\rho}\left(\mathbf{r}^{\prime}\right)\right) d \mathbf{r} d \mathbf{r}^{\prime},
$$

where $\rho$ is the non-expanded density, which, for closedshell systems can be written in terms of the occupied molecular orbitals $\phi_{i}$ as $\rho=2 \sum_{i}\left|\phi_{i}\right|^{2}$. As we have done in Ref.10, we can enforce charge conservation by including the following constraint:

$$
\Delta^{\mathrm{Q}}=\lambda\left(\int \tilde{\rho}(\mathbf{r}) d \mathbf{r}-N\right)^{2},
$$

where $N$ is the total number of electrons in the molecule and $\lambda$ is the weight given to the charge-conservation functional. We typically set $\lambda=1000$.

The expansion for the atomic density $\rho^{a}$ is given by

$$
\rho^{a}(\mathbf{r})=\sum_{k} c_{k}^{a} \xi_{k}^{a}(\mathbf{r}),
$$

where the $\xi_{k}^{a}$ are basis functions associated with site $a$ (these will normally be gaussian-type orbitals (GTOs) centred at $a$ ) and the coefficients $c_{k}^{a}$ are to be determined by minimizing an appropriate ISA functional. The basis set used for the atomic expansion of site $a$ will normally be a subset of the auxiliary basis used in the density fitting, limited to only include functions centered on site $a$. However, due to the the differences in the density-fitting and ISA functionals, we should expect to use different basis sets for each. The main reason for this is that the density fitting is performed using the entire monomer auxiliary basis set: basis deficiencies at a site can be somewhat made up for using auxiliary functions from neighbouring sites. This flexibility is not present in the ISA functional. Additionally, as we shall see, the ISA functionals require considerable flexibility in the AIM density tails.

Given an atomic density $\rho^{a}$ expanded in a basis of spherical GTOs, the atomic shape function $w^{a}$ is trivially defined as just the $s$-function $(l=0)$ part of $\rho^{a}$. That is, with the above expansion for $\rho^{a}$ we get

$$
w^{a}(\mathbf{r})=\sum_{k \in \mathrm{s} \text {-func }} c_{k}^{a} \xi_{k, \mathrm{~s}}^{a}(\mathbf{r}),
$$

where we have emphasised the $s$-character of the expansion functions with the additional ' $\mathrm{s}$ ' in the subscript. In this manner, the ISA spherical average step, eq. [2], is trivial when using basis expansions for the atomic functions. By contrast, it is cumbersome to implement on a grid.

Recently Verstraelen et al. [9] have described a similar approach which they call the Gaussian-ISA, or GISA, method. The GISA method is formally exactly the same as the Lillestolen-Wheatley ISA except that the shape functions are described using an expansion in a series of s-functions as is proposed here. However our proposal differs from the GISA method in important ways, which we will detail below.

The basis-space implementation of the stockholder partitioning step in eq. (11) is not unique and can be achieved using a variety of functionals. The obvious choice is to minimize

$$
\Delta_{\text {stock(A) }}=\sum_{a}\left\|\left(\rho^{a}-\rho \frac{w^{a}}{\sum_{b} w^{b}}\right)^{2}\right\|,
$$


where $\|\cdots\|$ indicates an appropriate norm, which in this case must be the overlap norm, as the integrand must be evaluated numerically on a grid. The following alternative allows us to use either the coulomb or overlap norm:

$$
\Delta_{\text {stock(B) }}=\sum_{a}\left\|\left(\rho^{a} \sum_{b} w^{b}-\rho w^{a}\right)^{2}\right\| .
$$

Notice that both functionals allow us to determine the atomic densities $\rho^{a}$ one at a time. This possibility can be used to make the algorithm scale linearly with the number of atoms.

\section{A. A naïve ISA algorithm}

It might seem that a straightforward ISA algorithm would be:

1. Initialize the shape functions $w^{a}$. While the starting point does not matter, a good starting point will ensure faster convergence. Reasonable choices are:

(a) Set the coefficients of one GTO to be 1.0 and the rest zero.

(b) Use the density-fitting solution to determine the starting coefficients.

2. Determine all the atomic densities $\rho^{a}$ using either $\Delta_{\text {stock(A) }}$ or $\Delta_{\text {stock(B) }}$. This can be done one atom at a time.

3. Update the shape functions $w^{a}$.

4. Check for convergence (see below). If not converged, iterate.

This algorithm can lead to converged shape functions, but when we use the standard density-fitting basis sets to describe the ISA atomic densities, the converged $\Delta_{\text {stock(A/B) }}$ is non-zero, and the total charge is incorrect by around 0.001 to 0.01 electrons. The primary cause for this discrepancy is that there is not enough flexibility in the typical density fitting auxiliary basis sets to fit the total density and the ISA atomic tails simultaneously. As explained above, in a standard fit to the density using eq. (4), this lack of flexibility is not an issue as we minimize the functional $\Delta^{\mathrm{DF}}$ in the variational space spanned by the molecular auxiliary basis set. By contrast, in the ISA procedure described above, the fit to the density of site $a$ is performed using only basis functions at that site. This significantly reduces the variational flexibility of the basis, and hence results in a poor fit to the total density.

This problem is resolved by an increase in the auxiliary basis flexibility, but this in turn leads to numerical instabilities. These are first manifested in small negative terms in the tail regions of the AIM densities $\rho^{a}$, which, as the näive ISA iterations progress, tend to grow uncontrollably and lead to a meaningless solution. (The proof of convergence of the ISA method[8] fails if the weight function becomes negative anywhere.) In the following we will describe how the basis sets are extended, and instabilities controlled, while simultaneously retaining the linearity of the ISA functionals.

\section{B. ISA basis sets}

The main problem with the auxiliary basis sets designed for density-fitting is that their s-function block is not flexible enough to describe the ISA atomic density tails well enough. They need to be described very well for the ISA solution to stabilize and converge reliably. There may also be inadequacies in the higher angular functions, and we have some evidence that this may be the case, but it is the s-functions that are the most important, due to their role in determining the AIM shape functions. We have therefore created hybrid 'DF-ISA' auxiliary basis sets that comprise a very flexible s-function set designed to allow good modelling of the ISA shape-function tails, together with the higher symmetry functions from the standard RI-MP2 density fitting basis sets [11, 12].

There are a few requirements for a good ISA basis: it should be flexible enough to be able to describe the ISA atomic shapes (particularly in the region of the tail), but it should lead to well-behaved linear equations. If the basis is too flexible, we encounter instabilities in the ISA functionals, and if the basis is too small, we find that total charge is not conserved by 0.01 electrons or more in the minimization of the ISA functionals (eqns. (8) and (97)). Indeed, for a good basis we not only see faster convergence and charge conservation to $10^{-3}$ electrons or better, but also find that the shape functions are positive everywhere with well-defined exponential tails. These criteria can be used as a means of assessing the quality of the ISA basis sets.

We have found that a reasonable choice for the ISA sfunction basis set is to use an even-tempered set with exponents of the form $\alpha=2^{n}$ a.u., $n=n_{\min } \ldots n_{\max }$, where $n_{\max }$ is 5 for hydrogen atoms $\left(\alpha_{\max }=32.0\right)$ and 8 for the heavier atoms $\left(\alpha_{\max }=256.0\right)$. For numerical stability we usually choose $n_{\min }=-3\left(\alpha_{\min }=0.125\right)$. While this choice results in well-behaved ISA shape functions for most systems, there are cases for which the 0.125 exponent for hydrogen atoms needs to be omitted, while for silicon an exponent of 0.0625 was added. We have termed this set as 'ISA/set2'. With this ISA basis set, the $\Delta_{\text {stock(A) }}$ functional conserves charge to $10^{-3}$ electrons or better.

While the above procedure works well for the ISA shape functions $w^{a}$, as mentioned above, it may be that the higher angular momentum functions in the densityfitting sets also need to be augmented to better model the ISA atomic densities $\rho^{a}$. We are investigating this possibility. 


\section{Fixing the shape-function tail}

The ISA shape functions are generally well-behaved in the core density region, but often exhibit problems in the tail. As mentioned above, negative terms in the expansion can lead to catastrophic instabilities and need to be controlled. Furthermore, as there is very little weight given to the small densities of the tail region, solutions can easily emerge which have odd features for low densities (less than about $10^{-5}$ a.u.) Effects of this order are small, and may have no apparent adverse effect on the overall ISA solution, but such behaviour is unsatisfactory.

The first ingredient needed to improve the tail is suggested by the empirical observation, from calculations on a variety of systems, that well-converged shape functions tend to exhibit an atom-like exponential decay. We therefore require all ISA shape functions to decay in this manner; that is, $w^{a}$, and hence the ISA atomic density $\rho^{a}$, should decay exponentially as

$$
w_{\mathrm{L}}^{a}(\mathbf{r})=A_{a} \exp \left(-\alpha_{a}\left|\mathbf{r}-\mathbf{R}_{a}\right|\right),
$$

where $\mathbf{R}_{a}$ is the centre of atom $a$, and $A_{a}$ and $\alpha_{a}$ are constants yet to be determined. We note here that although the more accurate decay of an atomic density is $A r^{\beta} \exp (-\alpha r)$, we have found that the additional polynomial factor does not appear to be important, and in the interest of simplicity it is omitted.

We now define the corrected shape function $\tilde{w}^{a}$ as

$$
\tilde{w}^{a}(\mathbf{r})= \begin{cases}w^{a}(\mathbf{r}) & \text { if }|\mathbf{r}| \leq r_{0}^{a} \\ w_{\mathrm{L}}^{a}(\mathbf{r}) & \text { otherwise. }\end{cases}
$$

Here $r_{0}^{a}$ is a distance up to which we may expect the uncorrected shape function $w^{a}(\mathbf{r})$ to be reliable. We typically take this to be a fixed multiple (usually 1.5 ) of the Slater radius [13] of the atom $a$, though in principle this radius could be determined self-consistently by examining the manner in which $w^{a}$ decays. The constants $A_{a}$ and $\alpha_{a}$ in $w_{\mathrm{L}}^{a}$ are determined by requiring continuity at $r_{0}$ and by ensuring that the charge contained in $\tilde{w}^{a}$ is identical with the charge in $w^{a}$. Both conditions can be enforced analytically. The charge conservation condition is necessary; without it the corrected shape functions $\tilde{w}^{a}$ alter the site charges by a small amount at each step, causing a slow divergence of the ISA iterations.

We now use the corrected shape functions in the ISA functionals given in eqns. (8) and (9). Notice that $\tilde{w}^{a}$ is not an expansion in GTOs, but, due to its piece-wise continuous form, must instead be defined numerically on a grid. Consequently, if used in the functional $\Delta_{\text {stock(A) }}$, this functional must use the overlap norm, as it is not practical to evaluate a six dimensional coulomb integral using grids.

To further stabilize the ISA atomic tails we increase the weights given to the tail by using, instead of the overlap norm, the following tail-weighted norm:

$$
\|f\|_{\text {tail }}=\int f(\mathbf{r}) \exp \left(+\epsilon\left|\mathbf{r}-\mathbf{R}_{a}\right|^{2}\right) d \mathbf{r},
$$

where $\epsilon$ is a positive number that must be less than twice the smallest exponent in the basis set so as to ensure integrability. We apply this weight to the s-function block only.

There is a degree of self-consistency in this process as all the parameters in the tail correction are updated at each iteration. Additionally, the shape function tailweighting is only applied when the shape function tails have been determined to be sufficiently stabilized. Finally, both the tail correction and the additional weighting can be removed in the final iterations if a fully selfconsistent ISA solution is required.

\section{A Robust ISA algorithm}

The improvements described above significantly improve the stability and accuracy of the basis-space ISA (BS-ISA) procedure, but we still see small charge violations (of the order of $10^{-3}$ electrons) when minimizing the functionals $\Delta_{\text {stock(A/B) }}$. These are very likely linked to the still insufficiently flexible ISA basis sets and possibly also to the nature of the fix applied to the shapefunction tails. While these charge violations are typically small, we need a method which will guarantee a good fit to the density while obtaining the best ISA solution possible within the basis set constraints. This is possible by simultaneously minimizing the $\Delta^{\mathrm{DF}}$ and $\Delta^{\mathrm{Q}}$ functionals together with either $\Delta_{\text {stock(A) }}$ or $\Delta_{\text {stock(B) }}$. We will define the BS-ISA functional using a single parameter $\zeta \in[0,1]$ to control the relative weights of the density-fitting and ISA functionals as follows:

$$
\Delta_{\mathrm{DF}-\mathrm{ISA}}(\zeta)=(1-\zeta)\left(\Delta^{\mathrm{DF}}+\Delta^{\mathrm{Q}}\right)+\zeta \Delta^{\text {stock(A/B) }} .
$$

Notice that we have included the charge conservation functional $\Delta^{\mathrm{Q}}$ with the density-fitting functional. This was done primarily for convenience of implementation; ideally it might be desirable to include $\Delta^{\mathrm{Q}}$ without a $\zeta$ dependence. In the discussion that follows, $\Delta_{\text {DF-ISA }}(\zeta)$ will mean the variant using $\Delta_{\text {stock(A) }}$ as most of our results have been obtained with this choice.

Ideally we would want our results to be independent of the choice of $\zeta$, and, indeed, for well-converged systems we will show that the dependence on $\zeta$ is small. This parameter controls the off-diagonal blocks that allow basis functions on neighbouring sites to be used to model the density at a given site. For $\zeta$ near 1, the diagonal blocks are dominant and we obtain a solution close to the pure ISA solution, while for $\zeta$ near 0 , the off-diagonal blocks are large and we achieve a more accurate fit to the total density, though with a relaxation of the ISA atomic densities. In practice, for a good BS-ISA basis set, values of 
$\zeta$ between 0.1 and 0.9 appear to be satisfactory, with very little variation in the final results.

We now describe a robust version of the basis-space ISA method:

1. Initialize the shape functions $w_{n=0}^{a}$ as described in the naïve ISA algorithm presented in sec. II A.

2. Attempt to determine the corrected form of the shape-function tails given in eq. (11). This is not always possible, and if the parameters of the function $w_{\mathrm{L}}^{a}$ are deemed to be unphysical, the fix is not attempted.

3. Minimize the $\Delta_{\mathrm{DF}-\mathrm{ISA}}(\zeta)$ functional to obtain the ISA atomic densities $\rho^{a}$.

4. Update the shape functions $w_{n+1}^{a}$ using the ISA atomic densities $\rho^{a}$ (eq. (7)).

5. Check convergence by evaluating:

$$
d^{a}=\frac{\left\langle w_{n}^{a} \mid w_{n+1}^{a}\right\rangle}{\sqrt{\left\langle w_{n}^{a} \mid w_{n}^{a}\right\rangle\left\langle w_{n+1}^{a} \mid w_{n+1}^{a}\right\rangle}} .
$$

With a convergence parameter $\epsilon$ (typically $10^{-9}$ ), we have achieved convergence if $\left|1-d^{a}\right| \leq \epsilon \forall a$.

\section{If converged, exit.}

7. If the shape functions are deemed to be sufficiently stabilized, turn on the additional tail weighting (eq. (12)). This is usually done if convergence is attained to $\tilde{\epsilon}=10^{-5}$.

\section{Iterate from step 2.}

Because this algorithm combines density-fitting and the BS-ISA methods, we will refer to this as the BS-ISA+DF algorithm.

We mention here that there are many variants of the BS-ISA+DF algorithm: We could, for example, use the $\Delta_{\mathrm{DF}-\mathrm{ISA}}(\zeta)$ functional with a fixed value of $\zeta$ until convergence is attained. Alternatively, we could minimize $\Delta_{\mathrm{DF}-\mathrm{ISA}}(\zeta=1.0)$ so as to converge the shape functionals, and only then reduce $\zeta$ to fit the total density better. This variant has the advantage that $\zeta=1.0$ corresponds to minimizing the $\Delta_{\text {stock(A) }}$ functional only, and this can be made to scale linearly with the number of atoms. Consequently, this approach may be better suited to large systems. Once convergence has been attained, with $\zeta<1.0$, the off-diagonal blocks in the DF-ISA matrices are nonzero and allow the solution to relax to fit the total density better, though with a slight degradation of the ISA solution.

\section{E. Relation to the GISA variant}

As noted above, Verstraelen et al. [9] have recently described an analogous approach that they call the Gaussian-ISA, or GISA, method. It is formally exactly the same as the Lillestolen-Wheatley ISA except that the shape functions are described using an expansion in a series of s-functions as proposed here. Our proposal differs from the GISA method in important ways: firstly, we use a far more complete set of s-functions which are essential for adequate convergence of the shape-function tails. We find that this flexibility is needed to ensure that functional $\Delta_{\text {stock(A) }}$ conserves charge to $10^{-3}$ electrons or better (without application of the global charge conservation constraint). As we have noted above, the better the basis set, the better charge is conserved, as the functional is able to satisfy the ISA conditions and reproduce the total density simultaneously. With the GISA basis sets we see charge violations of between 0.01 (formamide) and 0.2 (benzene) while the ISA/set2 results in significantly smaller charge violations of just 0.001 to 0.003 electrons, clearly indicating that the ISA/set2 basis possesses the variational flexibility needed to accurately describe the ISA shape functions.

Of course, charge conservation is not an issue if the stockholder partitioning (eq. (1)) is performed in real space, as is done by Verstraelen et al. and in the original formulation by Lillestolen and Wheatley. In that case, as long as the shape functions are positive everywhere and finite in extent, no matter how pathological they might otherwise be, charge will always be conserved. From our experience, and the results of Verstraelen et al., the shape functions obtained using the GISA basis sets can be reasonable, but we find that they often exhibit pathologies in the way the tails decay. One such example is illustrated in fig. 1 for the benzene molecule (aug-cc-pVTZ basis). The shape functions have been obtained by minimizing $\Delta_{\text {stock(A) }}$ using the GISA and ISA/set2 basis sets with all other parameters the same. The ISA/set2 atomic shapes exhibit a clear exponential decay (in the range shown in the figure) which contrasts with the somewhat erratic and non-exponential decay of the shape functions obtained using the GISA basis sets.

Finally we point out that our approach results in linear equations that are readily suited for applications to large molecular systems and that, because our ISA s-function basis sets are created using a simple algorithm, the basis sets can be extended to other atomic systems with relatively little effort.

\section{F. BS-ISA+DF numerical details and implementation}

Minimizing the density-fitting and chargeconservation functionals, eqns. (4) and (5), leads to 


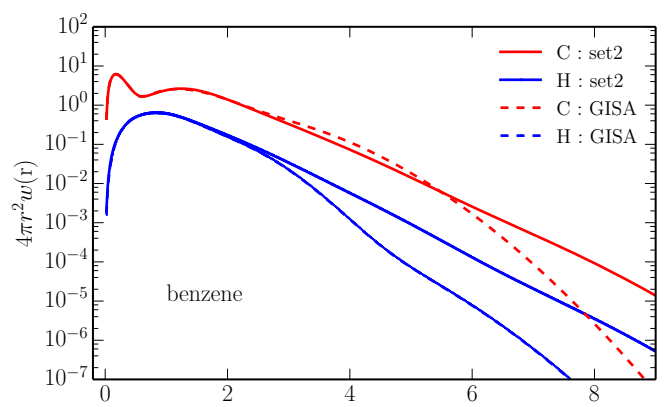

FIG. 1: Shape functions for the carbon and hydrogen atoms in benzene (aug-cc-pVTZ basis) calculated using the GISA basis sets and the ISA/set 2 basis set described in this paper. In both cases functional $\Delta_{\text {stock(A) }}$ was minimized.

the following linear equations:

$$
S_{k, k^{\prime}}^{\mathrm{DF}} d_{k^{\prime}}=T_{k}^{\mathrm{DF}},
$$

where $\mathbf{d}$ is the coefficient vector for the density expansion given in eq. (3), and the L.H.S. matrix $\mathbf{S}^{\mathrm{DF}}$ and R.H.S. vector $\mathbf{T}^{\mathrm{DF}}$ are defined as

$$
\begin{aligned}
S_{k, k^{\prime}}^{\mathrm{DF}} & =\left\langle k \| k^{\prime}\right\rangle+\lambda I_{k} I_{k^{\prime}} \\
T_{k}^{\mathrm{DF}} & =\langle\rho \| k\rangle+\lambda N I_{k},
\end{aligned}
$$

where $\left\langle k \| k^{\prime}\right\rangle$ signifies the coulomb integral of the basis functions $\chi_{k}$ and $\chi_{k^{\prime}}$, and $I_{k}=\int \chi_{k}(\mathbf{r}) d \mathbf{r}$. The $\mathbf{S}$ matrix is order $N \times N$, where $N$ is the number of auxiliary basis functions in the system. Therefore the computational cost of solving eq. 15p scales as $O\left(N^{3}\right)$. On the other hand, the functionals $\Delta_{\text {stock(A) }}$ and $\Delta_{\text {stock(B) }}$ are both expressed as the sum over sites of functionals that depend on the auxiliary basis functions located on the site only. This allows us to perform the minimization of these functionals piecewise, with $O(N)$ computational cost, by minimizing one block at a time. For example, $\Delta_{\text {stock(A) }}$ can be written as

$$
\begin{aligned}
\Delta_{\text {stock(A) }} & =\sum_{a}\left\|\left(\rho^{a}-\rho \frac{w^{a}}{\sum_{b} w^{b}}\right)^{2}\right\|, \\
& =\sum_{a} \Delta_{\text {stock(A) }}^{a} .
\end{aligned}
$$

The minimization of $\Delta_{\text {stock(A) }}^{a}$ leads to the equations

$$
\tilde{S}_{k, k^{\prime}}^{a} d_{k^{\prime}}^{a}=\tilde{T}_{k}^{a},
$$

where the superscript $a$ indicates that the quantities defined here depend only on functions centered at site $a$, and

$$
\begin{aligned}
\tilde{S}_{k, k^{\prime}}^{a} & =\left\langle k \| k^{\prime}\right\rangle \\
\tilde{T}_{k}^{a} & =\int \chi_{k}^{a}(\mathbf{r}) \rho(\mathbf{r}) \frac{w^{a}(\mathbf{r})}{\sum_{b} w^{b}(\mathbf{r})} d \mathbf{r} .
\end{aligned}
$$

Notice that when considered as a matrix over all sites $a$, the $\tilde{\mathbf{S}}$ matrix is block-diagonal.

For each site $a$, the solution of eq. (19) involves a computational cost of order $O\left(N^{0}\right)$. As there are $O(N)$ sites, the total computational cost of minimizing $\Delta_{\text {stock(A) }}$ scales as $O(N)$. However to achieve this linear scaling the $\tilde{T}_{k}^{a}$ integrals must be calculated using locality. In CAMCASP we did this as follows:

- Neighbours are defined for every site. A site $b$ is considered a neighbour of site $a$ if the overlap integral of the most diffuse auxiliary basis function (all functions are treated as s-functions for this purpose) of sites $a$ and $b$ exceeds a specified threshold. This is a reasonable definition as density-fitting is used for all quantities in the CAMCASP program.

- The integration grid used in eq. (21) is constructed from the atom grids of site $a$ and neighbouring sites only.

- Likewise, the density-fitted molecular density $\rho$ and pro-molecular density $\sum_{b} w^{b}(\mathbf{r})$ are evaluated using auxiliary basis functions located on the site $a$ and its neighbours only.

With these considerations, $\tilde{T}_{k}^{a}$ can be evaluated with computational cost of order $O\left(N^{0}\right)$.

The linear equations for the BS-ISA+DF functional in eq. (13) can be obtained by constructing the ISA equations for the full molecular system from the atomic site equations in eq. (19), and combining this set of equations with the density-fitting equations in eq. (15) to give

$$
\mathcal{S}_{k, k^{\prime}} d_{k^{\prime}}=\mathcal{T}_{k},
$$

where if $k \in a$ and $k^{\prime} \in a^{\prime}$ then

$$
\begin{aligned}
\mathcal{S}_{k, k^{\prime}} & =(1-\zeta) S_{k, k^{\prime}}^{\mathrm{DF}}+\zeta\left(\sum_{b} \delta_{b a} \delta_{b a^{\prime}} \tilde{S}_{k, k^{\prime}}^{b}\right) \\
\mathcal{T}_{k} & =(1-\zeta) T_{k}^{\mathrm{DF}}+\zeta\left(\sum_{b} \delta_{b a} \tilde{T}_{k}^{a}\right) .
\end{aligned}
$$

These equations can be solved exactly like the standard density-fitting equations.

\section{BS-ISA+DF SHAPE FUNCTIONS AND CONVERGENCE}

The BS-ISA+DF calculations reported in this paper have been performed using atomic densities calculated using the PBE0 [14] functional, asymptotically corrected using the Fermi-Amaldi [15] correction and the Tozer \& Handy [16] splicing scheme. Density functional calculations have been performed using the DALTON 2.0 program [17] using a patch included with the SAPT2008 [18] 
program. Unless otherwise specified, we have used the Dunning aug-cc-pVQZ basis [19, 20] for density functional calculations. Vertical ionisation potentials needed for the asymptotic correction have either been calculated using the $\Delta$-DFT algorithm or have been taken from the NIST Chemistry Web-book [21].

The BS-ISA+DF algorithm described here has been implemented in a pre-release version of the CAMCASP program [22] and is available from the authors upon request. Iso-density maps reported in this paper have also been calculated with CAMCASP and are displayed using the ORIENT program [23]. All multipole models have been calculated using the CAMCASP program.

We have investigated a number of systems including $\mathrm{H}_{2}, \mathrm{H}_{2} \mathrm{O}, \mathrm{CH}_{4}, \mathrm{CCl}_{4}, \mathrm{NH}_{3}, \mathrm{LiF}, \mathrm{H}_{2} \mathrm{CO}, \mathrm{CO}, \mathrm{CO}_{2}$, pyridine (d-aug-cc-pVTZ basis), benzene (aug-cc-pVTZ basis), formamide, $\mathrm{HF}$ and $\mathrm{C}_{10} \mathrm{~N}_{2} \mathrm{H}_{13}$ (aug-cc-pVDZ basis), but will report only a subset of the data in this paper.

\section{A. Convergence}

The biggest problem associated with the ISA algorithm has been its poor convergence properties. Realspace algorithms can take more than a thousand iterations to converge or may not converge at all [9]. The GISA algorithm of Verstraelen et al. fares far better with algorithmic convergence in 140 iterations or so. However, due to the restricted variational flexibility of the GISA basis sets, this is not true convergence, as we have pointed out above.

There are no such issues with the BS-ISA+DF algorithm, which we have found to converge in at most 80 iterations and sometimes as few as 10 , without any convergence acceleration techniques that might be applicable to the algorithm. There is no apparent effect of system size on the number of iterations required for convergence, though we have noticed that basis set improvements can lead to even faster convergence, and conversely, a small or unbalanced basis can lead to poor convergence. In fig. 2 we display convergence patterns for the BS-ISA+DF algorithm for a representative sample of the systems we have investigated. Our normal convergence criterion is that all shape functions converge to $10^{-9}$ or better using eq. (14), but for illustrative purposes we have chosen a threshold of $10^{-12}$ for the pyridine molecule (density obtained with the d-aug-cc-pVTZ basis). Plotted together with max $\left|1-d^{a}\right|$ are the ISA charges of the heavier atoms of pyridine. The hydrogen atoms are omitted for clarity. The charges can be seen to converge very smoothly, but $\max \left|1-d^{a}\right|$ exhibits oscillations that die off just before the $40^{\text {th }}$ iteration, only to re-appear and subsequently die off again. This is quite a common occurrence; it can be seen for the $\mathrm{NH}_{3}$ system too, and we have no explanation for this behaviour.

The LiF molecule shows the fastest convergence of any we have studied, with smooth and rapid convergence in 12 iterations. While the same is true for $\mathrm{CCl}_{4}$, here we observed oscillations in the ISA site charges. These die off by iteration 25 . The convergence patterns for the other systems we have studied fall into one of these four categories and are not presented here.

Notice that in all cases, past a threshold, convergence is exponentially fast with iteration number. This seems to be true in general. Additionally, there is no apparent relation between rate of convergence and system size: the relatively small ammonia molecule took 80 iterations to converge, but the largest system we have investigated ( 25 atoms) took 53 iterations. This is particularly promising as this is a desirable property for large applications.

\section{B. Shape Functions}

In fig. 3 we report shape functions for the atoms in the pyridine, formamide, $\mathrm{LiF}$ and $\mathrm{CCl}_{4}$ systems. Rather than plot $w(r)$ directly, we have plotted $4 \pi r^{2} w(r)$ to better illustrate the shell structure of the atoms in these systems. All shape functions have been calculated with the BSISA+DF algorithm with $\zeta=0.9$. This value of $\zeta$ was chosen as shape functions are generally better behaved for $\zeta$ closer to 1, when the $\Delta_{\text {stock(B) }}$ is dominant.

The pyridine molecule illustrates the success of the BS-ISA+DF method. All atomic shape functions are well-behaved, with clear exponential tails. This is not always the case. For the formamide molecule we were able to obtain shape functions that were positive everywhere only after eliminating the most diffuse $(\alpha=0.125) \mathrm{s}$ function from the ISA basis set for the hydrogen atoms. This explains why the $\mathrm{H} 1$ and $\mathrm{H} 2$ hydrogen shape functions of formamide decay quickly past 6 Bohr. Despite these changes, the shape function for the oxygen AIM appears to be somewhat spurious past 6 Bohr. Very small changes to the shape function expansions are responsible for this kind of non-exponential decay, and they occur in regions where the density is so small that, even with the schemes described in sec. IIC, it can sometimes be difficult to control the behaviour of the shape functions.

The shape functions for the LiF molecule clearly pick out the substantial differences between the $\mathrm{Li}$ and $\mathrm{F}$ atoms in LiF. In principle, the lithium AIM should determine the density decay of the system and, indeed, it seems to have the slower decay until about 6 Bohr when the decay of both atoms become similar. This is a consequence of the basis set used. Both atoms use the same ISA s-function basis set, so at sufficiently long-range their shape functions must decay in a similar manner.

Also shown in fig. 3 are shape functions for carbon atoms in different molecules. The ISA charges on these carbon atoms are -0.11 (benzene), -0.42 (methane), and $+0.51\left(\mathrm{CCl}_{4}\right)$. This is what would be expected from the electronegativity differences of the bonding atoms. The 

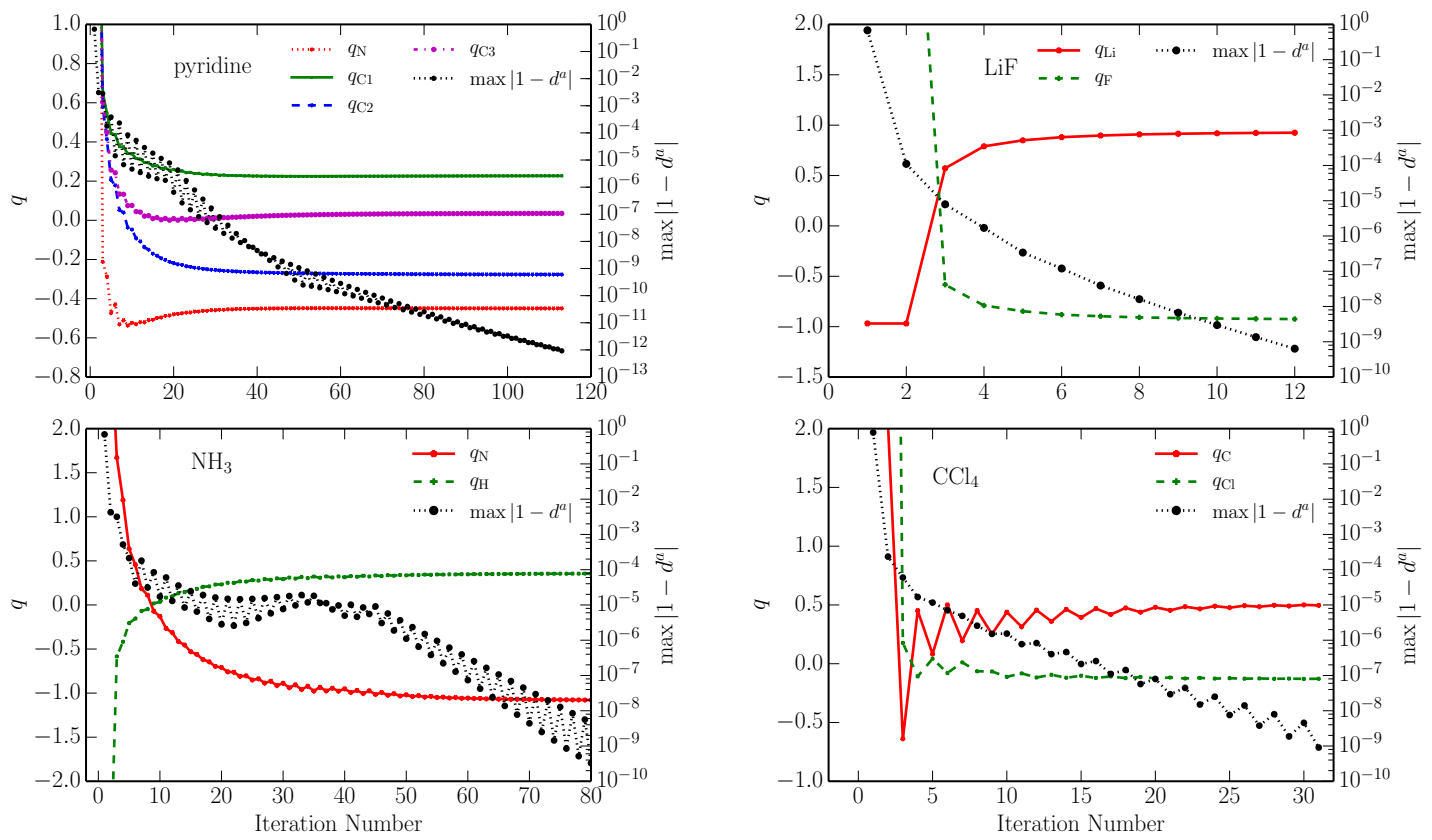

FIG. 2: BS-ISA convergence patterns for pyridine, $\mathrm{LiF}, \mathrm{NH}_{3}$ and $\mathrm{CCl}_{4}$. We have plotted the AIM charges (left $y$-axis) and convergence parameter $\max \left|1-d^{a}\right|$ (right $y$-axis; see eq. (14)) against iteration number. Most calculations use a convergence threshold of $10^{-9}$, but for pyridine we have reduced the threshold to $10^{-12}$ to better illustrate the convergence properties of the BS-ISA algorithm. The pyridine density was calculated using the d-aug-cc-pVTZ basis while for the the other molecules the aug-cc-pVQZ basis was used.
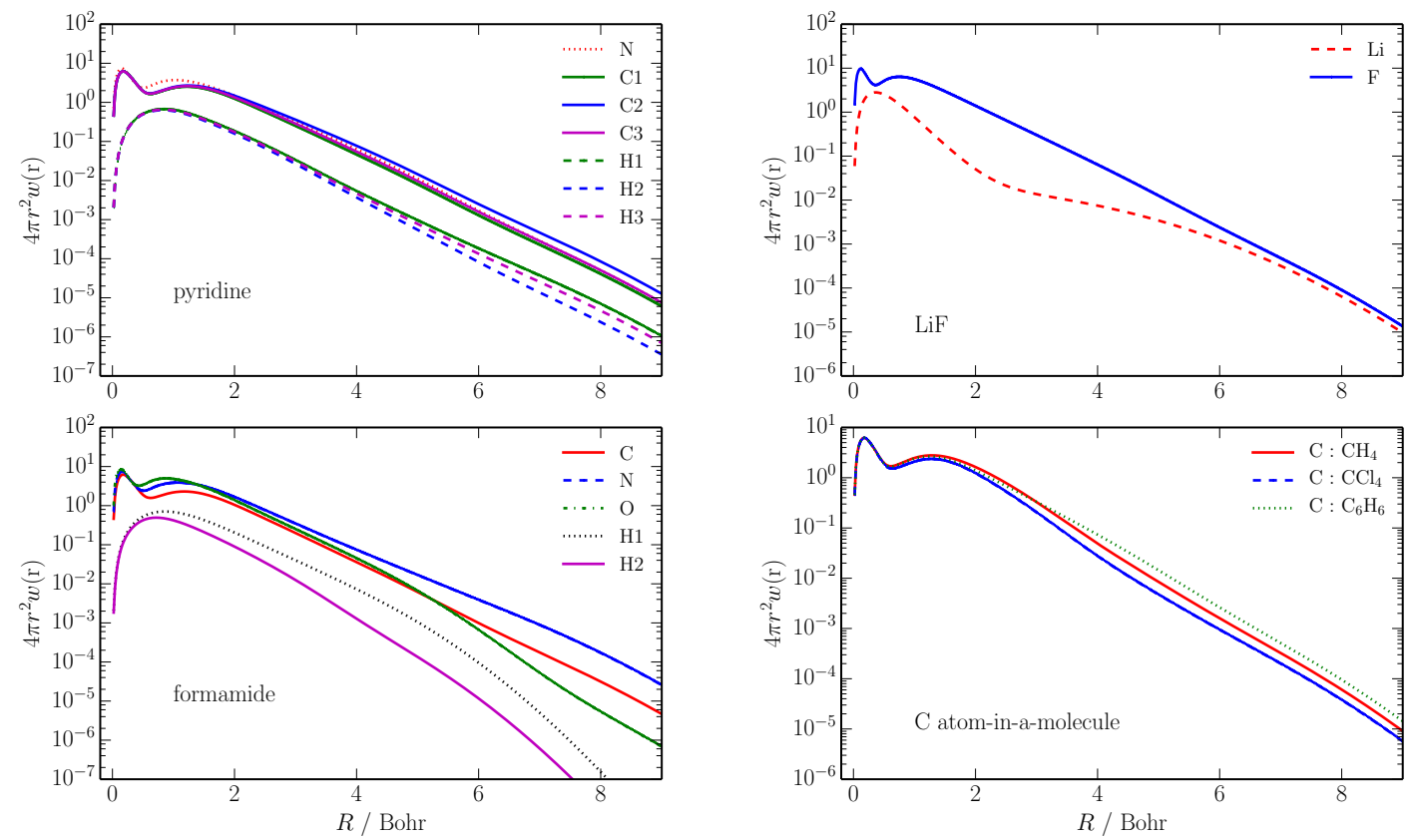

FIG. 3: BS-ISA+DF shape functions for atoms in pyridine, $\mathrm{LiF}$ and formamide. We have plotted $4 \pi r^{2} w(r)$ to better illustrate the shell structure. The aug-cc-pVQZ basis was used to calculate the density, except for pyridine, where d-aug-cc-pVTZ was used. The BS-ISA+DF calculation was performed using the aQZ/set2 basis, but for formamide we had to limit the $s$-functions on the hydrogen atoms to a smallest exponent of 0.25 a.u. The amide hydrogen atoms in formamide are very similar, and for clarity only one is shown. In the last panel carbon shape functions are compared for carbon atoms in benzene, methane and carbon tetrachloride. All shape functions were obtained using the BS-ISA+DF algorithm with $\zeta=0.9$. 


\begin{tabular}{lcccccc}
\hline Rank: & 0 & 1 & 2 & 3 & 4 & 5 \\
\hline$D M A O$ & & & & & & \\
$\langle\Delta\rangle$ & -1.63 & 0.44 & 0.95 & 0.46 & 0.39 & 0.36 \\
$\sigma_{\Delta}$ & 205.45 & 40.36 & 14.93 & 3.73 & 1.03 & 0.79 \\
$D M A 4$ & & & & & & \\
$\langle\Delta\rangle$ & -1.15 & -4.29 & -0.55 & 0.02 & 0.07 & 0.08 \\
$\sigma_{\Delta}$ & 53.32 & 98.98 & 11.37 & 2.48 & 1.20 & 0.82 \\
$B S-I S A+D F$, & $\zeta$ & $=0.1$ & & & & \\
$\langle\Delta\rangle$ & -0.89 & -0.49 & -0.41 & 0.21 & 0.20 & \\
$\sigma_{\Delta}$ & 33.91 & 18.62 & 14.52 & 3.62 & 3.14 & \\
\hline
\end{tabular}

TABLE I: Table of differences between the electrostatic potential calculated using CAMCASP (SAPT-DFT) and various multipole models over a surface at twice the van der Waals radii of the atoms in the formamide molecule. Values are in millivolt $(\mathrm{mV}) .\langle\Delta\rangle=\left\langle E_{\text {elst }}^{(1)}-E_{\text {elst }}^{(1)}[\mathrm{DM}]\right\rangle$ is the mean difference and $\sigma_{\Delta}$ is the standard deviation. See the text for details.

\begin{tabular}{lrrrrrr}
\hline Rank: & \multicolumn{1}{c}{0} & 1 & 2 & 3 & 4 & 5 \\
\hline DMAO & & & & & & \\
$\langle\Delta\rangle$ & 4.0 & 10.5 & 12.3 & 9.8 & 9.6 & 9.5 \\
$\sigma_{\Delta}$ & 322.9 & 88.5 & 42.5 & 11.5 & 4.9 & 4.6 \\
$D M A 4$ & & & & & & \\
$\langle\Delta\rangle$ & 5.8 & 4.9 & 6.2 & 8.7 & 8.9 & 9.0 \\
$\sigma_{\Delta}$ & 108.2 & 199.3 & 32.2 & 10.5 & 6.9 & 5.3 \\
$B S-I S A+D F$, & $\zeta=0.1$ & & & & \\
$\langle\Delta\rangle$ & 6.0 & 7.2 & 7.3 & 8.9 & 8.9 & \\
$\sigma_{\Delta}$ & 64.2 & 38.9 & 34.2 & 10.0 & 8.3 \\
\hline
\end{tabular}

TABLE II: Table of differences between the electrostatic potential calculated using CAMCASP (SAPT-DFT) and various multipole models over a surface at 1.5 times the van der Waals radii of the atoms in the formamide molecule. Values are in millivolt $(\mathrm{mV})$. See caption to table $\Pi$ for details.

BS-ISA+DF algorithm correctly shows that the carbon AIM density in methane is more diffuse than the carbon in carbon tetrachloride. The carbon atom in benzene is more diffuse than the methane and $\mathrm{CCl}_{4}$ carbons due to the planar nature of the benzene molecule.

\section{MULTIPOLES}

The BS-ISA+DF method offers us a computationally efficient and numerically robust implementation of the ISA method. In the first paper on the ISA method, Lillestolen and Wheatley noted that the ISA charges were remarkably good at describing the molecular dipole moments, often better than the DMA method. Of course the charges alone are not the whole story and one needs to take into account the higher ranking multipole moments too. This was recognised by Stone [24-26] and it is the presence of these higher ranking terms that are the main reason for the success of both the 1985 and 2005 DMA algorithms [27, 28]. Consequently, a meaningful assessment of multipole moment models must include a com-

\begin{tabular}{lrrrr}
\hline Rank: & 0 & 1 & 2 & 4 \\
\hline$D M A O$ & & & \\
$\langle\Delta\rangle$ & +78.8 & +92.2 & +95.4 & +86.0 \\
$\sigma_{\Delta}$ & 496.5 & 200.0 & 121.3 & 30.0 \\
$D M A 4$ & & & \\
$\langle\Delta\rangle$ & +82.9 & +44.6 & +74.6 & +84.0 \\
$\sigma_{\Delta}$ & 243.6 & 408.4 & 114.0 & 47.7 \\
$B S-I S A+D F, \zeta=0.1$ & & \\
$\langle\Delta\rangle$ & +79.8 & +82.9 & +82.9 & +85.0 \\
$\sigma_{\Delta}$ & 132.7 & 84.0 & 87.1 & 40.4 \\
\hline
\end{tabular}

TABLE III: Table of differences between the electrostatic potential calculated using CAMCASP (SAPT-DFT) and various multipole models for the formamide molecule over an isodensity surface at an electron density of 0.001 a.u. Values are in millivolt $(\mathrm{mV})$. See caption to table $\mathrm{I}$ for details.

parison of the higher ranking terms. But these are not unique, and this poses a problem: how are we to assess one model against another?

At distances well outside the van der Waals surface of the molecule, we can compare the electrostatic potential calculated from the multipole expansions - we will denote these as $E_{\text {elst }}^{(1)}[\mathrm{DM}]$, the 'DM' to indicate the distributed multipole description - with the reference nonexpanded potential, obtained from SAPT(DFT) as the energy $E_{\text {elst }}^{(1)}$ of a unit charge. The latter includes the penetration energy, which is absent in $E_{\text {elst }}^{(1)}[\mathrm{DM}]$, but at large distances it will be small.

Table II shows the mean difference, $\langle\Delta\rangle=\left\langle E_{\text {elst }}^{(1)}-\right.$ $\left.E_{\text {elst }}^{(1)}[\mathrm{DM}]\right\rangle$, between reference and model electrostatic potentials at points on the surface of formamide at twice the van der Waals radii, for various multipole models, and the standard deviation, $\sigma_{\Delta}$, of these differences. The columns show results for models truncated at different ranks; that is, 'rank $n$ ' means that multipoles up to rank $n$ are included and higher multipoles discarded. For a good model, the mean should be small but nonzero, representing the mean penetration energy, and the standard deviation $\sigma_{\Delta}$ which represents the fluctuation in penetration energy over the surface, should also be small. The values are in millivolt; the energy of a unit charge is numerically the same value in meV. The penetration energy here should be positive, as the positive test charge is less screened from the nuclei as it penetrates into the electron density.

The Table shows two variants of distributed multipole analysis. DMA0 is the original version[24] and DMA4 is the modified version[26] which uses numerical integration over a grid for terms in the gaussian expansion of the density with exponents $\zeta$ less than 4 . Finally the ISA results were obtained using the BS-ISA+DF method described above.

Distributed multipole analysis is not recommended for use at low rank, as local dipoles enter at rank 1, and 

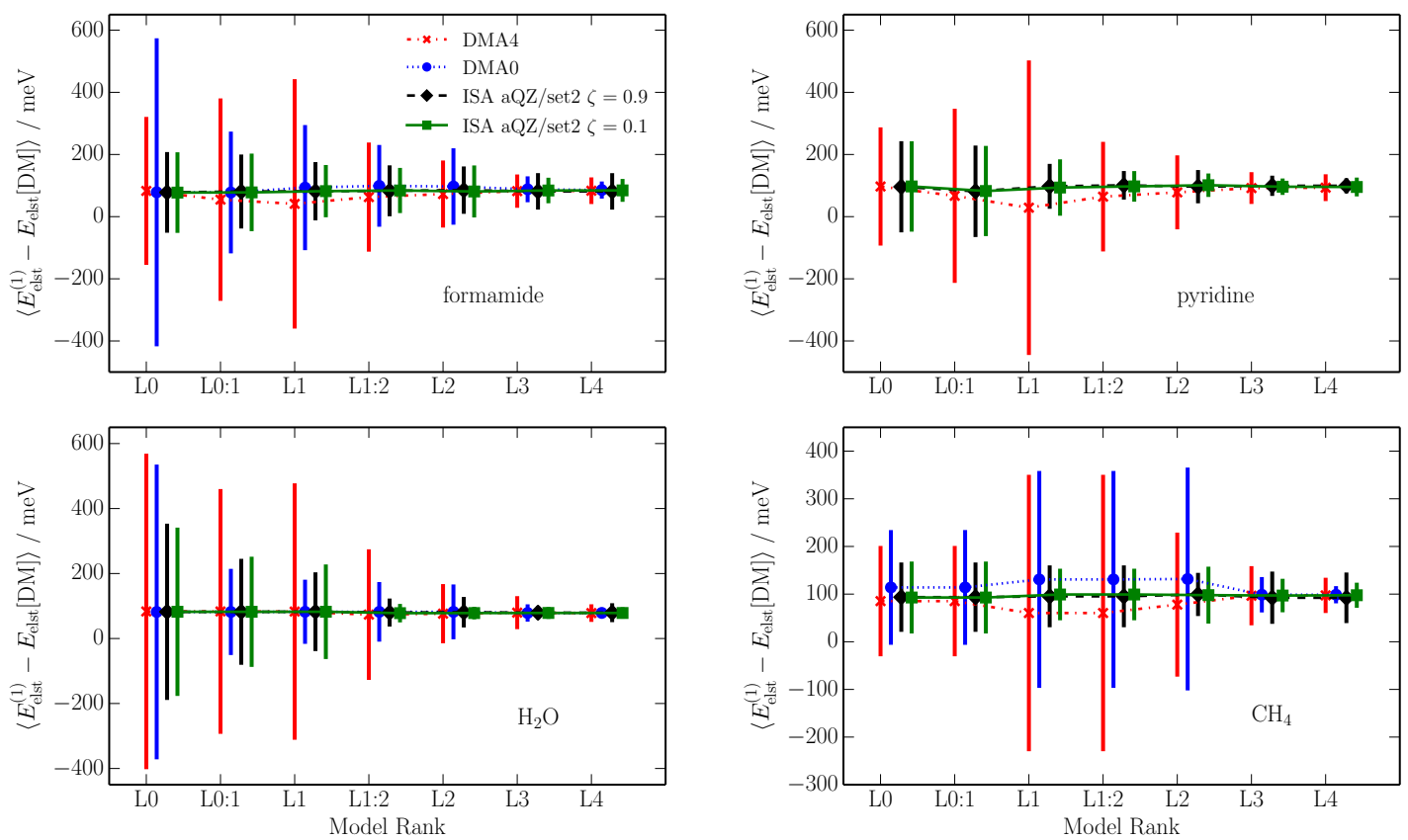

FIG. 4: Average signed potential differences and and their standard deviation calculated using a point charge probe placed on the $10^{-3}$ iso-surfaces of the pyridine, water, formamide and methane molecules. The DMA0, DMA4 and BS-ISA $\zeta=0.1,0.9$ models at various ranks are used to calculate the multipole energies. Ranks are indicated by either $\mathrm{L} n$ or $\mathrm{L} n: m$. In the former case, all atoms have multipoles limited to rank $n$ and in the latter, hydrogen atoms are limited to rank $n$ and heavier atoms to rank $m$.

quadrupoles, describing $\pi$-orbital features, at rank 2 , and the results at rank 0 and 1 are poor, as expected. At rank 2 and above, however, it performs well, and the DMA0 and DMA4 variants are comparable. It is clear, however, that the BS-ISA+DF results are substantially better at low rank, and comparable with the DMA results at rank 2 and above.

Table I shows similar results for a surface at 1.5 times the van der Waals radii. The variation about the mean is much greater here, but the general picture is the same. The mean energy difference is now greater, but seems to be quite well described by the ISA model at quite low rank.

Table III shows results on an isodensity surface around formamide at an electron density of 0.001 a.u. Results at rank 3 are similar to rank 4 and are not shown. The mean differences are larger here, as expected, and they vary with rank for DMA0 and DMA4, but are remarkably consistent for BS-ISA+DF. Moreover the standard deviation of the differences is noticeably smaller for the ISA method, which suggests that it may provide a promising approach for modelling the penetration energy, and we discuss this further below.

The data are more clearly displayed in fig. 4, where $\langle\Delta\rangle$ and $\sigma_{\Delta}$ are plotted versus model. For a multipole model with good convergence properties the average energy difference $\langle\Delta\rangle$ from the SAPT(DFT) reference will converge quickly with rank, and the standard deviation $\sigma_{\Delta}$ of the differences will be small. From fig. 4 it can be seen that the two DMA models appear to have converged only when terms up to rank 3 are included. By contrast, with the BS-ISA+DF multipole models the average energy difference has converged by rank 1 (charge and dipole) though $\sigma_{\Delta}$ reduces to an acceptable value only by rank 3 , as with the DMA models. The BS-ISA+DF L0 model has a standard deviation $\sigma_{\Delta}$ of only $133 \mathrm{mV}$, about $10-15 \%$ more than the DMA0 and DMA4 L2 models. It would seem that, for this system, the L0 BS-ISA+DF multipole models are competitive with the more elaborate, higher rank models. This is also evident in fig. 4 where we see that both the BS-ISA+DF models (with $\zeta=0.1$ and 0.9) result in energy differences that show very little variation with rank, though, for higher accuracies we need to include terms of rank 3 on to reduce the variation over the surface.

These formaldehyde data are also shown in the Supplementary Information as colour maps of $\Delta$ plotted on the 0.001 a.u. isosurface of formamide. The superior convergence pattern of the multipoles from the BS-ISA+DF method is clearly visible.

In considering these results, it should be borne in mind that these figures give penetration energies for a unit charge penetrating to the 0.001 a.u. isodensity surface, which is approximately the van der Waals surface. The corresponding energy, on these figures, is of the order of $80 \mathrm{meV}$, or about $8 \mathrm{~kJ} / \mathrm{mol}$. In a real system, it is the elec- 
trons of each molecule that penetrate into the other, and the charge involved is much smaller, by a factor of at least 10 , for each atom-atom interaction, so the penetration energies will also be much smaller.

In fig. 4 we display similar data for three other systems: water, pyridine and methane. The DMA0 models are not included for pyridine as the energy differences obtained with this model are too large to be meaningfully displayed along with energies from the other models. The broad conclusions reached with the formamide system are seen to hold for all molecules: the BS-ISA+DF models (with $\zeta=0.1$ and 0.9 ) result in energy differences which exhibit the fastest convergence with rank and the smallest variation over the surface. On the other hand, the DMA0 and DMA4 models show considerably more erratic average energy differences and significantly larger values of $\sigma_{\Delta}$. However, the L3 and L4 models from all four methods tend to be reasonably close, with similar values of $\langle\Delta\rangle$ and $\sigma_{\Delta}$.

\section{A. Assessing the models using molecular dimers}

From the discussion above it should be clear that the BS-ISA+DF multipole models show better convergence behaviour than the DMA models. It is also evident from the data presented in 4 that the BS-ISA+DF point charge (L0) models are better than those from both DMA algorithms. This is not unexpected, as the ISA algorithm guarantees the most spherical atomic domains (within precision and algorithmic implementation), and the DMA method does not claim to produce useful charge models. However a pertinent question is whether these L0 models with charges on atomic sites only can be used for modelling the electrostatic interaction, and what kinds of error should we expect if this is done. This issue is particularly pertinent as many simulation programs are not able to use anything other than point charges, and, in any case, for large simulations involving biologically interesting molecules, it is often not feasible to use higher ranking multipoles due to the computational cost incurred. To fully address the questions associated with point charge models would take us too far from the central aim of this paper, so we just outline the issues. Further, from fig 4 it may seem that the DMA0, DMA4 and two BS-ISA+DF models are nearly equivalent when high ranking multipoles are included: the L3 and L4 models from these methods appear very similar. However these results were obtained using a point-charge probe interacting with the molecule and some differences between the models are not picked up in this way.

We now address these issues using energies calculated for the water, pyridine and methane dimers at a variety of orientations. In figs. 5, 6 and 7 we display, for the DMA0, DMA4 and BS-ISA+DF $(\zeta=0.1)$ multipole models at various ranks, the difference between the SAPT(DFT) electrostatic energy and energies calculated using the models. For brevity, we refer to this difference as the 'multipole error'. It includes deficiencies in the multipole model as well as the penetration energy. Since it is usually assumed that the penetration energy is proportional to the first-order exchange energy, the energy differences are plotted against the first-order exchange energy $E_{\text {exch }}^{(1)}$, and we expect a straight line if the assumption is correct and if the multipole model is good enough. Plotting the results in this way also illustrates the smaller energies and the differences between the models more clearly. Note that, for convenience we plot $-\Delta=-\left(E_{\mathrm{elst}}^{(1)}-E_{\mathrm{elst}}^{(1)}[\mathrm{DM}]\right)$ against $E_{\mathrm{exch}}^{(1)}$ in these figures.

First of all consider the data for the water dimer. In fig. 5 we plot $\Delta$ against $E_{\text {exch }}^{(1)}$ for 400 pseudo-random water dimer configurations generated using the CAMCASP program [22, 29]. From fig. 5] we see that all three L0 (point charge) models show a very poor correlation between $\Delta$ and $E_{\text {exch }}^{(1)}$. This should not be a surprise as a considerable body of work has shown that to model the electrostatic energy accurately in small molecules like water, additional charges are needed on off-atomic sites. For example, most accurate models of water use five sites for the electrostatic model (see for example the SAPT-5s potential[30]). The correlation between $\Delta$ and $E_{\text {exch }}^{(1)}$ improves only when we include terms to rank 2 on all sites. At all ranks, the BS-ISA+DF multipoles result in the best correlation of $\Delta$ and $E_{\text {exch }}^{(1)}$ with the correlation growing as rank increases. At rank 4 the DMA0 and BS-ISA+DF models result in similar energies, but the DMA4 model exhibits somewhat more scatter. Since $\Delta$ approaches the penetration energy as the rank of the model increases, we may state that there is an approximate correlation between the penetration energy and the first-order exchange energy. This has been observed before, but there does not appear to be a proof of this correlation.

The relationship between the penetration energy (here taken to be the L4 $\Delta$ energies) and $E_{\text {exch }}^{(1)}$ appears to be linear for a fixed dimer orientation, but the constant of proportionality is dependent on the dimer geometry. The orientational dependence of this proportionality is illustrated in the last panel (at rank 4) in fig. 5 where we include data for dimers in the hydrogen-bonded configuration of water. The BS-ISA+DF and DMA0 models both result in similar $\Delta$ energies that show a linear correlation with $E_{\text {exch }}^{(1)}$, but with a different constant of proportionality compared with the high-energy configurations from the pseudo-random data set. The DMA4 model once again yields the worst correlation and may not be suitable for the water molecule.

A consequence of the above observations is that we should expect the best correlation between the penetration energy and $E_{\text {exch }}^{(1)}$ for systems that are close to spherical, such as the methane molecule. In fig. 6 we plot $\Delta$ against $E_{\text {exch }}^{(1)}$ for the methane dimer in 2600 pseudo- 

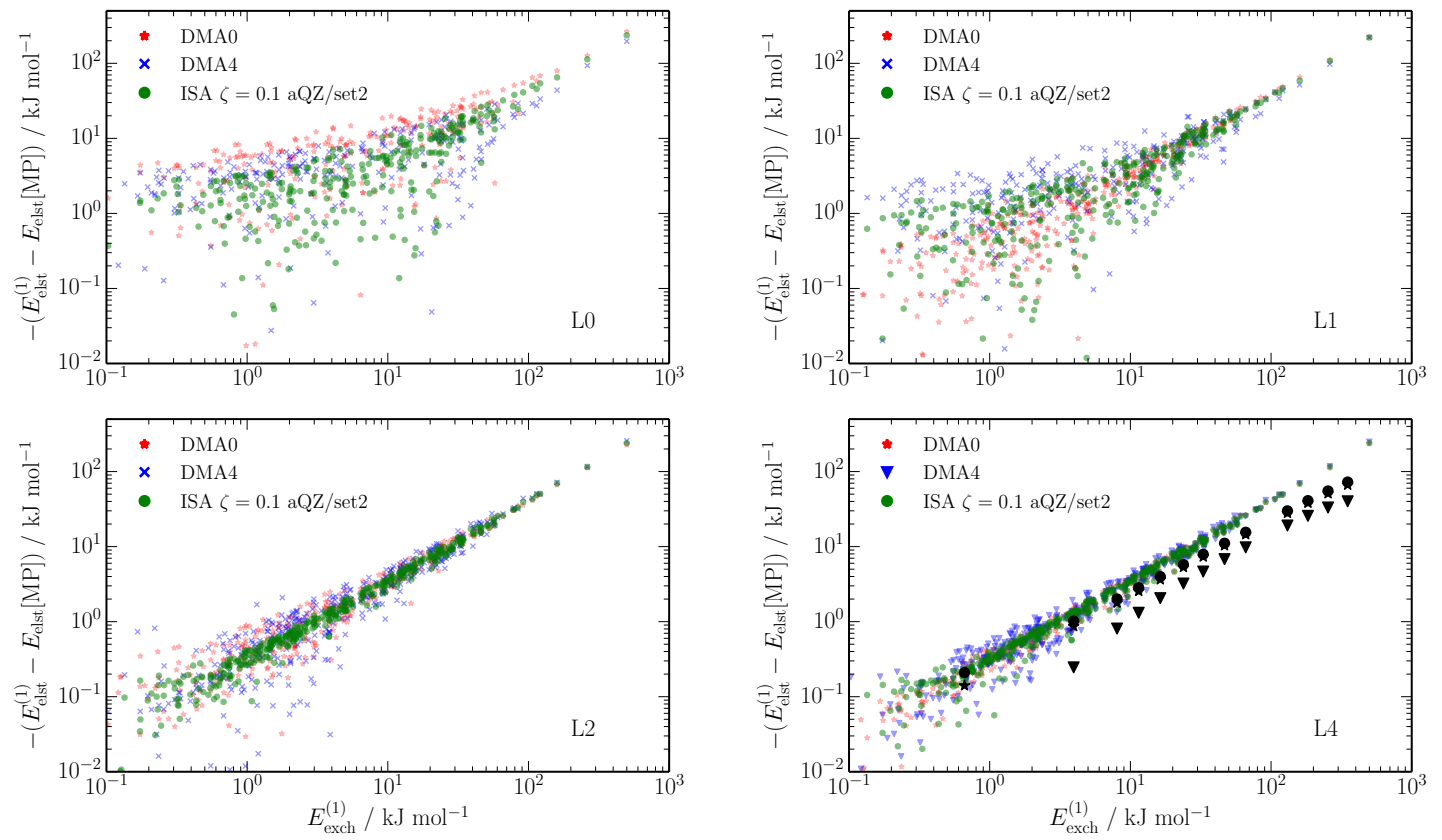

FIG. 5: Multipole errors for the water dimer in 400 pseudo-random dimer geometries. The multipole error is plotted against the firstorder exchange-repulsion $E_{\text {exch. }}^{(1)}$. The multipole models have been calculated using the aug-cc-pVQZ main basis and the SAPT(DFT) $E_{\mathrm{elst}}^{(1)}$ and $E_{\mathrm{exch}}^{(1)}$ energies have been calculated using the aug-cc-pVTZ basis in the MC+ type. See the text for details. In the rank 4 panel we have additionally included data (large black points) for the water dimer in its minimum energy dimer orientation.
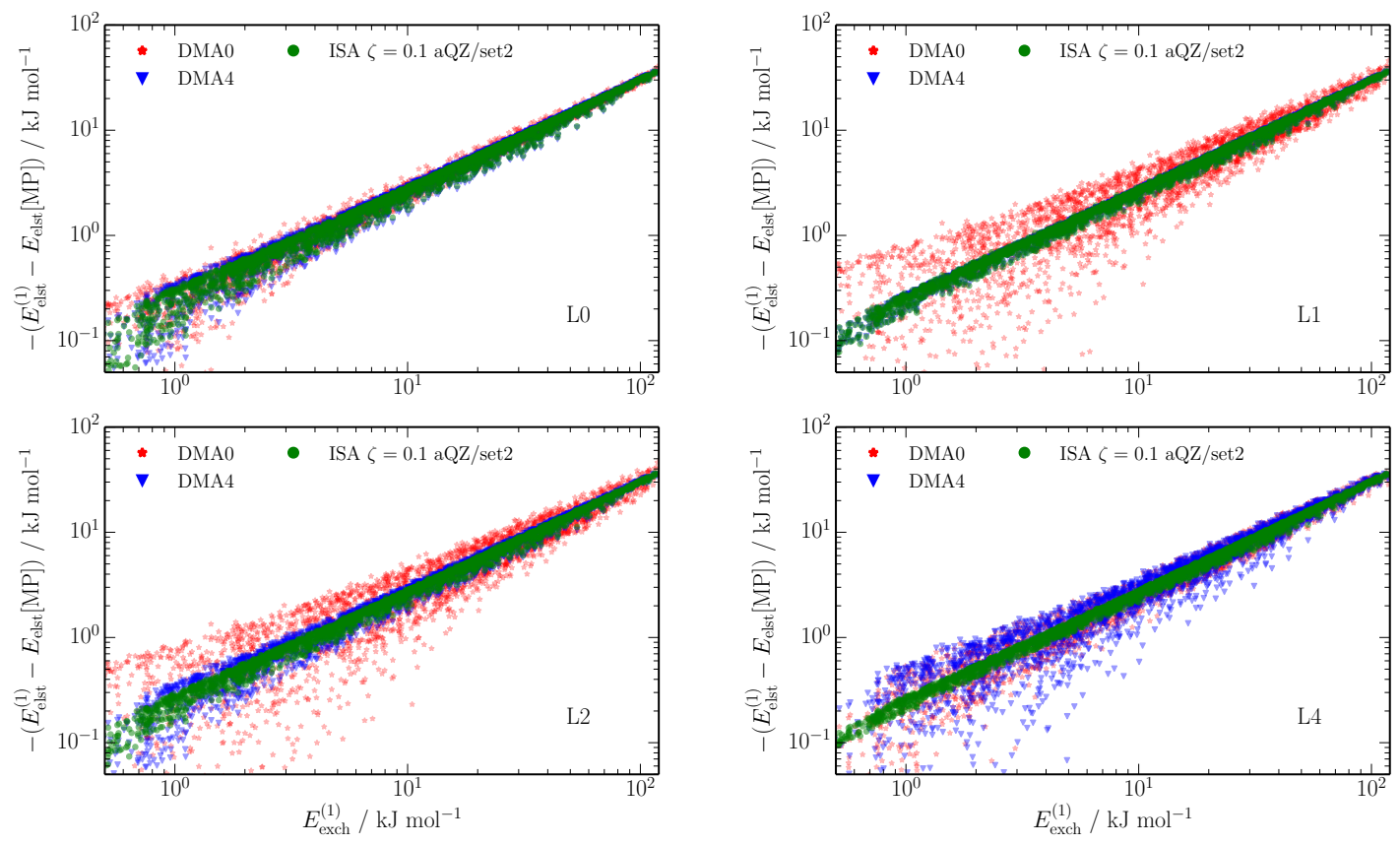

FIG. 6: Multipole errors for the methane dimer in 2600 pseudo-random dimer geometries. The multipole error is plotted against the first-order exchange-repulsion $E_{\text {exch }}^{(1)}$. The multipole models have been calculated using the aug-cc-pVQZ main basis and the SAPT(DFT) $E_{\mathrm{elst}}^{(1)}$ and $E_{\mathrm{exch}}^{(1)}$ energies have been calculated using the aug-cc-pVTZ basis in the MC+ type. See the text for details. 

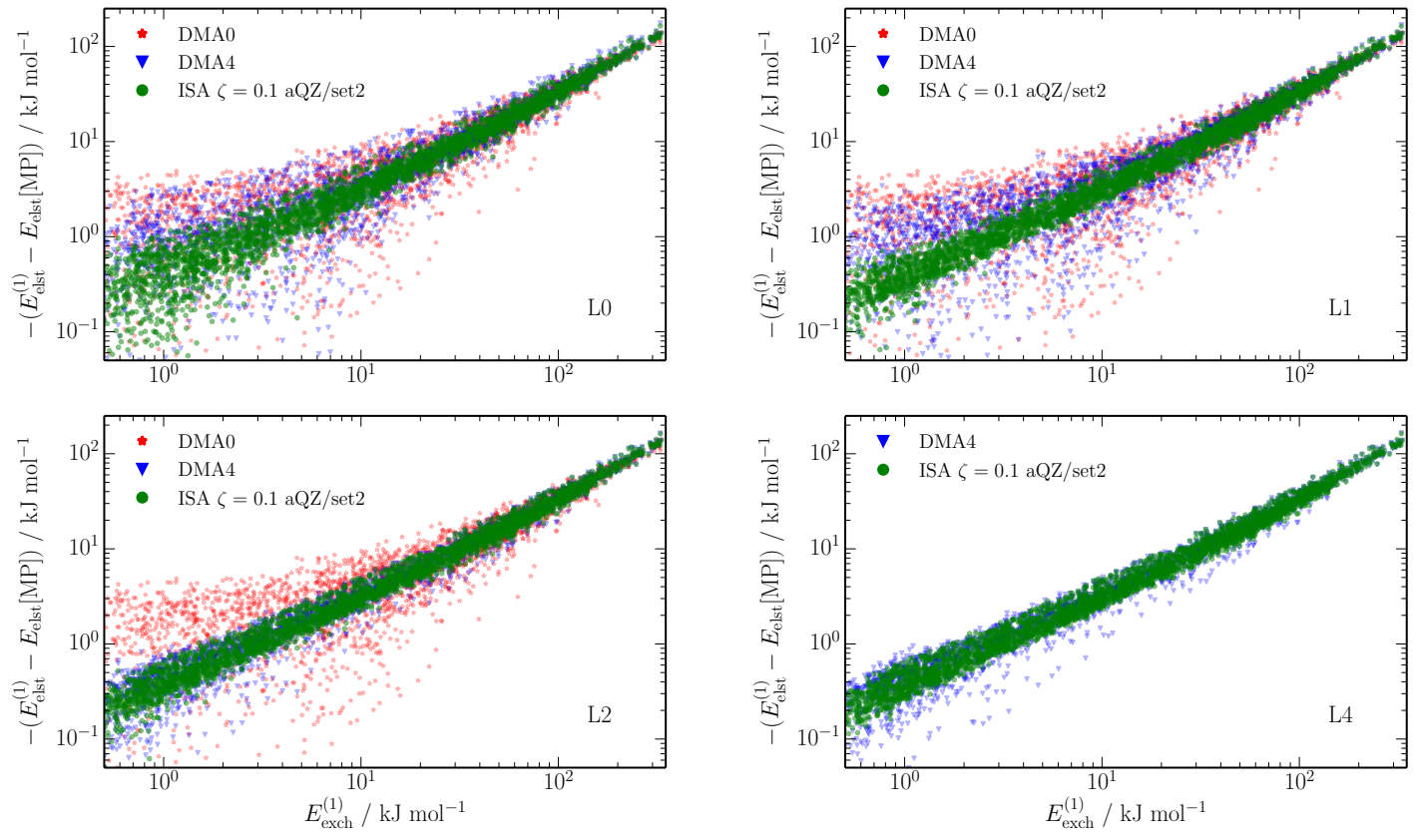

FIG. 7: Multipole errors for the pyridine dimer in pseudo-random dimer geometries. The multipole error is plotted against the firstorder exchange-repulsion $E_{\text {exch }}^{(1)}$. The multipole models have been calculated using the aug-cc-pVQZ main basis and the SAPT(DFT) $E_{\text {elst }}^{(1)}$ and $E_{\text {exch }}^{(1)}$ energies have been calculated using the Sadlej pVTZ basis in the MC type. The scatter in the DMA0 penetration energies does not change with rank, consequently these results are not shown in the rank 4 panel so as to better highlight the DMA4 penetration energies. 
random configurations. Two features stand out: the BSISA+DF multipole model results in a strong correlation between $\Delta$ and $E_{\text {exch }}^{(1)}$ at all ranks, but by rank 4 (rank 3 results are similar) the correlation is nearly perfect. On the other hand, while the DMA0 and DMA4 models are comparable to the BS-ISA+DF model at rank 0 , there is no systematic behaviour of these models at higher ranks: the L1 DMA4 and BS-ISA+DF models are nearly identical but when terms beyond rank 2 are included, $\Delta$ from both DMA models is poorly correlated with $E_{\text {exch }}^{(1)}$. We note here that it is possible to improve the quality of the DMA4 multipoles by reducing the value of the Becke smoothening parameter [26] used to perform the realspace partitioning of the most diffuse functions, but it is not clear if this strategy can be expected to work more generally for other systems.

Finally, in fig. 7 we plot similar data for the pyridine dimer in around 3000 orientations. Pyridine is a highly anisotropic system so we should expect a strong orientational dependence in the proportionality between $\Delta$ and $E_{\text {exch }}^{(1)}$. This does seem to be the case. The correlation between these two energies is not as good as either the water dimer or the methane dimer, nevertheless, here too, it is the BS-ISA+DF model that yields the best correlation between $\Delta$ and $E_{\text {exch }}^{(1)}$ at all ranks. The DMA0 multipoles are poor at all ranks and the DMA4 model is a considerable improvement, though even this model cannot compete with the BS-ISA+DF, even when terms to rank 4 are included. Notice that once again the BS-ISA+DF model exhibits the fastest convergence with rank: the charge only model may be adequate for many purposes and we see nearly converged results when terms of rank 1 (dipoles) are included. The BS-ISA+DF model is essentially fully converged by rank 2 . These observations are in-line with those made from the data plotted in fig. 4.

\section{ANALYSIS}

We have described and presented results from a numerically stable and robust implementation of the iterated stockholder atoms (ISA) approach of Lillestolen and Wheatley [8]. This approach, termed the BS-ISA+DF method, works entirely in basis-space and can be combined with standard density-fitting functionals using a single parameter $\zeta$ that controls the relative weights of the BS-ISA and density-fitting functionals.

The BS-ISA+DF method uses auxiliary basis sets that are substantially larger that those normally used for density-fitting. In particular, the s-functions sets which are needed to define the ISA shape functions are unusually over-complete and flexible. This is needed as a considerable degree of variational flexibility is required to minimize the ISA part of the BS-ISA+DF functional. We have demonstrated that with smaller, more inflexible basis sets, the functional minimum does not correspond to the true minimum of the ISA functional. In particular, the shape functions are not well defined in the tail region.

The BS-ISA+DF functional is shown to converge in less than 80 iterations and sometimes as few as 10. Convergence is exponential with iteration number and seems to be independent of the molecular size or type of basis used. In contrast, conventional methods for solving the ISA equations either work in real-space and converge (if at all) in 1000 iterations or so, or partially work in an excessively restricted basis-space and converge to a false minimum in 140 iterations or so.

The numerical implementation of the BS-ISA+DF functional is identical with that of conventional densityfitting functionals, so it can easily be applied to systems of hundreds of atoms. Additionally, the pure ISA part of the BS-ISA+DF functional scales linearly with the number of atoms. This feature, and the overall high accuracy and good convergence properties of the BS-ISA+DF functional should make it ideally suited for applications to large molecules. One restriction, though, is that since we rely on Gaussian (finite-extent) basis sets, this functional cannot as yet be used with infinite systems.

The main goal of this paper has been to investigate the applicability of the ISA method as an alternative for distributed multipoles. Having a stable ISA implementation was essential for this as both the low and high ranking distributed multipoles are sensitive to the partitioning method, particularly to the way in which the atom-like density tails are modelled. This motivation was central to the attention we have paid to converging the atomic density tails in the BS-ISA+DF functional.

We have used the BS-ISA+DF method to calculate distributed multipoles and have compared electrostatic energies and multipole errors computed with these multipoles and those from distributed multipole analysis; both the 1985 version that works entirely in basis-space [24] giving the DMA0 multipoles, and the 2005 version that works partially in real-space and has better stability with basis sets [26] giving the DMA4 multipoles. For the dozen systems we have studied the BS-ISA+DF multipoles are found to give uniformly better results at low rank than both the DMA0 and DMA4 models. That is, if multipoles are limited to low rank (below 3), the BSISA+DF models are found to result in better-converged electrostatic/penetration energies than either the DMA0 or DMA4 models. Further, while the best BS-ISA+DF results are obtained with $\zeta=0.1$, that is, $10 \%$ ISA and $90 \%$ density-fitting functionals, the variation with $\zeta$ is usually insignificant and decreases with an increase in the variational flexibility of the auxiliary basis sets used in the minimization of the BS-ISA+DF functional.

At rank 0 (charges only) the BS-ISA+DF models are the most accurate we have obtained. They are often substantially more accurate than the DMA models at rank 0, but it must be emphasized that the DMA method is explicitly not intended for developing point charge models. 
The BS-ISA+DF models have been shown to exhibit the fastest convergence with rank of the multipole expansion. Further, in contrast to the DMA0 and DMA4 models, the BS-ISA+DF multipoles converge systematically with increasing rank. Therefore, these models can be truncated to lower ranks without erratic increases in the errors incurred. Additionally, the penetration energy - defined as the difference in the non-expanded electrostatic energy $E_{\text {elst }}^{(1)}$ and the multipole energy calculated using converged BS-ISA+DF model-exhibits a uniformly excellent correlation with the first-order exchange energy $E_{\text {exch }}^{(1)}$. This property makes the BS-ISA+DF multipole models an excellent choice for building intermolecular potentials, as the penetration energy is often fitted together with the first-order exchange [29, 31].

The numerical superiority of the BS-ISA+DF distributed multipoles makes them potential replacements for the DMA0 and DMA4 multipoles, which have set the benchmark for accuracy for the last thirty years. This has been a high benchmark to surpass but there were already indications from Lillestolen and Wheatley's original paper that the ISA might surpass the DMA and we find that this appears to be the case. However the original DMA method has some advantages over both its successor, DMA4, and the BS-ISA+DF method: it is numerically exact and computationally simple and it is very fast, even for large molecules. Furthermore, both DMA methods allow the addition of off-atomic expansion centres, which are not possible in the ISA approach. These may be reasons enough to use the DMA methods for cer- tain applications, but from the evidence provided here we suggest that the ISA, particularly in the BS-ISA+DF implementation shown here, is better suited for highly accurate, rapidly convergent, and perhaps, even more physically appealing, distributed multipole expansions.

\section{ADDITIONAL INFORMATION}

All developments have been implemented in a developer's version of the CAMCASP 5.8 [22] program which may be obtained from the authors on request. The supplementary information (SI) contains additional data from the systems we have investigated but not included in this paper. Additional information about the basis sets used is also included in the SI.

\section{ACKNOWLEDGEMENTS}

AJM and FF would like to thank Queen Mary University of London for support and the Thomas Young Centre for a stimulating environment. AJM would like to thank Dr Richard Wheatley and Dr János Ángyán for helpful comments and Université de Lorraine for a visiting professorship during which part of this work was completed. We would like to thank Rory A. J. Gilmore for the SAPT(DFT) calculations on the methane dimer.
[1] A. J. Misquitta, J. Spencer, A. J. Stone, and A. Alavi, Phys. Rev. B 82, 075312 (2010).

[2] C. F. Guerra, J.-W. Handgraaf, E. J. Baerends, and F. M. Bickelhaupt, J. Comput. Chem. 25, 189 (2004).

[3] P. Bultinck, D. L. Cooper, and D. V. Neck, Phys. Chem. Chem. Phys. 11, 3424 (2009).

[4] R. Bader, Atoms in Molecules (Clarendon Press, Oxford, 1990).

[5] G. Jansen, C. Hattig, B. A. Hess, and J. G. Angyan, Mol. Phys. 88, 69 (1996).

[6] D. S. Kosov and P. L. A. Popelier, J. Phys. Chem. A 104, 7339 (2000).

[7] L. Joubert and P. L. A. Popelier, Mol. Phys. 100, 3357 (2002).

[8] T. C. Lillestolen and R. J. Wheatley, Chem. Commun. 2008, 5909 (2008).

[9] T. Verstraelen, P. Ayers, V. V. Speybroeck, and M. Waroquier, Chem. Phys. Lett. 545, 138 (2012).

[10] A. J. Misquitta and A. J. Stone, J. Chem. Phys. 124, 024111 (2006).

[11] F. Weigend, M. Haser, H. Patzelt, and R. Ahlrichs, Chem. Phys. Lett. 294, 143 (1998).

[12] F. Weigend, A. Kohn, and C. Hattig, J. Chem. Phys. 116,
3175 (2002).

[13] J. C. Slater, J. Chem. Phys. 41, 3199 (1964).

[14] C. Adamo and V. Barone, J. Chem. Phys. 110, 6158 (1999).

[15] E. Fermi and E. Amaldi, Le orbite $\infty$ s delgi elementi (Reale Accademia d'Italia, 1934), vol. 6(1), pp. 119-149.

[16] D. J. Tozer and N. C. Handy, J. Chem. Phys. 109, 10180 (1998).

[17] T. Helgaker, H. J. A. Jensen, P. Joergensen, J. Olsen, K. Ruud, H. Aagren, A. Auer, K. Bak, V. Bakken, O. Christiansen, et al., Dalton, a molecular electronic structure program, release 2.0 (2005), see http://www.kjemi.uio.no/software/dalton/dalton.html

[18] R. Bukowski, W. Cencek, P. Jankowski, B. Jeziorski, M. Jeziorska, V. Lotrich, S. Kucharski, A. J. Misquitta, R. Moszynski, K. Patkowski, et al., SAPT2008: an ab initio program for many-body symmetry-adapted perturbation theory calculations of intermolecular interaction energies, University of Delaware and University of Warsaw (2008), accessed: Oct 2013, URL http://www. physics.udel.edu/ szalewic/

[19] T. H. Dunning, Jr., J. Chem. Phys. 90, 1007 (1989).

[20] R. A. Kendall, T. H. Dunning, Jr., and R. J. Harrison, J. 
Chem. Phys. 96, 6796 (1992).

[21] S. G. Lias, Ionization energy evaluation in nist chemistry webbook, nist standard reference database number 69, eds. w. g. mallard and p. j. linstrom, gaithersburg, 2000 (http://webbook.nist.gov), accessed: Oct 2013.

[22] A. J. Misquitta and A. J. Stone, CAmCASP: a program for studying intermolecular interactions and for the calculation of molecular properties in distributed form, University of Cambridge (2013), accessed: July 2014, URL http://wwW-stone.ch.cam.ac.uk/programs. html\#CamCASP

[23] A. J. Stone, A. Dullweber, O. Engkvist, E. Fraschini, M. P. Hodges, A. W. Meredith, D. R. Nutt, P. L. A. Popelier, and D. J. Wales, Orient: a program for studying interactions between molecules, version 4.8, University of Cambridge (2013), accessed: Oct 2013, URL http://wWw-stone. ch.cam.ac.uk/programs.html\#0rient.
[24] A. J. Stone and M. Alderton, Mol. Phys. 56, 1047 (1985).

[25] A. J. Stone and M. Alderton, Mol. Phys. 100, 221 (2002).

[26] A. J. Stone, J. Chem. Theory Comput. 1, 1128 (2005).

[27] G. M. Day, W. D. S. Motherwell, and W. Jones, Cryst. Growth Des. 5, 1023 (2005).

[28] S. L. Price, M. Leslie, G. W. A. Welch, M. Habgood, L. S. Price, P. G. Karamertzanisc, and G. M. Day, Phys. Chem. Chem. Phys. (2010).

[29] A. J. Misquitta, G. W. A. Welch, A. J. Stone, and S. L. Price, Chem. Phys. Lett. 456, 105 (2008).

[30] E. M. Mas, R. Bukowski, K. Szalewicz, G. C. Groenenboom, P. E. S. Wormer, and A. van der Avoird, J. Chem. Phys. 113, 6687 (2000).

[31] A. J. Stone and A. J. Misquitta, Int. Revs. Phys. Chem. 26, 193 (2007). 